\title{
BMJ Open Intersectoral approaches and integrated services in achieving the right to health for refugees upon resettlement: a scoping review protocol
}

Dena Javadi, ${ }^{1}$ Etienne V Langlois, ${ }^{1}$ Shirley Ho, ${ }^{1}$ Peter Friberg, ${ }^{2,3}$ Göran Tomson ${ }^{2,4}$

To cite: Javadi D, Langlois EV, Ho S, et al. Intersectoral approaches and integrated services in achieving the right to health for refugees upon resettlement: a scoping review protocol. BMJ Open 2017;7:e016638. doi:10.1136/ bmjopen-2017-016638

- Prepublication history and additional material for this paper are available online. To view please visit the journal (http:// dx.doi.org/10.1136/bmjopen2017-016638).

Received 16 March 2017 Revised 12 June 2017 Accepted 13 July 2017

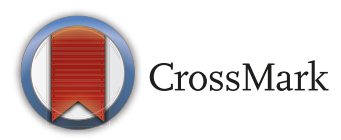

${ }^{1}$ Alliance for Health Policy and Systems Research, World Health Organization, Geneva, Switzerland

${ }^{2}$ Swedish Institute for Global Health Transformation (SIGHT), Royal Swedish Academy of Sciences, Geneva, Switzerland ${ }^{3}$ Department of Molecular and Clinical Medicine, Sahlgrens Academy and University Hospital, Gothenburg University, Gothenburg, Sweden

${ }^{4}$ Health System and Policy, Global Health, Karolinska Institutet, Stockholm, Sweden

Correspondence to

Dena Javadi; javadid@who.int, denajavadi@gmail.com

\section{ABSTRACT}

Introduction Global insecurity and climate change are exacerbating the need for improved management of refugee resettlement services. International standards hold states responsible for the protection of the right of non-citizens to an adequate standard of physical and mental health while recognising the importance of social determinants of health. However, programmes to protect refugees' right to health often lack coordination and monitoring. This paper describes the protocol for a scoping review to explore barriers and facilitators to the integration of health services for refugees; the content, process and actors involved in protecting refugee health; and the extent to which intersectoral approaches are leveraged to protect refugees' right to health on resettlement, especially for vulnerable groups such as women and children.

Methods and analysis Peer-reviewed (through four databases including MEDLINE, Web of Science, Global Health and PsycINF0) and grey literature were searched to identify programmes and interventions designed to promote refugee health in receiving countries. Two reviewers will screen articles and abstract data. Two frameworks for integration and intersectoral action will be applied to understand how and why certain approaches work while others do not and to identify the actors involved in achieving success at different levels of integration as defined by these frameworks.

Ethics and dissemination Findings from the scoping review will be shared in relevant conferences and meetings. A brief will be created with lessons learnt from successful programmes to inform decision making in design of refugee programmes and services. Ethical approval is not required as human subjects are not involved.

Trial registration number Registered on Open Science Framework at https://osf.io/gt9ck/.

\section{INTRODUCTION}

The Office of the United Nations High Commission for Human Rights identifies the right to the enjoyment of the highest attainable standard of physical and mental health as a fundamental part of human rights, first articulated in the 1946 Constitution of the WHO. ${ }^{1}$ While the right to health includes
Strengths and limitations of this study

- Many programmes exist to attempt to address the unique challenges faced by refugees, but these are largely unevaluated and face challenges to sustainability. This scoping review will summarise lessons learnt from these programmes by exploring barriers and facilitators to the integration of health services for refugees; the content, process and actors involved in protecting refugee health; and the extent to which intersectoral approaches are leveraged to protect refugees' right to health on resettlement, especially for vulnerable groups such as women and children.

- This study will be limited by the quality of the literature on healthcare programmes for refugees and protection of their right to health.

access to healthcare and the hard infrastructure associated with that, such as hospitals and ambulances, it also includes the underlying determinants of health, including safe drinking water, adequate sanitation, safe food, adequate nutrition and housing, healthy working and environmental conditions, health-related education and information and gender equality. ${ }^{1}$ Freedoms that protect individuals from non-consensual medical treatment, torture and other degrading treatment are also included in this definition. Furthermore, entitlements under the right to health include universal health coverage-now a target under Sustainable Development Goal (SDG) 3-broadly covering access to preventive and curative services, essential medicines, timely basic health services, health-related education and participation in health-related decision making at both national and community levels. ${ }^{12}$ Especially relevant to the plight of refugees, the right to health includes non-discrimination, whereby health services, goods and facilities must be provided to all without any discrimination. Lastly, these health services must be accessible, available in sufficient 
quantity, medically and culturally acceptable and of good quality, which includes having a trained health workforce, safe products and adequate sanitation. ${ }^{2}$

The influx of refugees over the last few years makes the realisation of these rights a legal and logistical challenge. ${ }^{34}$ Different in definition from the term 'migrant', 'refugees' are those fleeing armed conflict or persecution as defined by the 1951 Refugee Convention, which also identifies their basic rights, specifically that refugees should not be returned to situations that are deemed a threat to their life or freedom. ${ }^{5}$ A key distinction is that refugee rights are a matter of national legislation and of international law. ${ }^{6}$ Despite these legal protections, refugees face many challenges in accessing health services, especially more vulnerable groups like women and children. ${ }^{34}$ Many states explicitly exclude refugees from the level of protection afforded to their citizens, instead choosing to offer 'essential care' or 'emergency healthcare', which is differentially defined across countries. ${ }^{7}$ The Committee on the Elimination of Racial Discrimination and the Committee on Economic, Social and Cultural Rights both include general comments that hold States accountable to 'the right of non-citizens to an adequate standard of physical and mental health by, inter alia, refraining from denying or limiting their access to preventive, curative and palliative health services'. ${ }^{8}$

However, the capacity of States to truly protect these rights is limited. ${ }^{9}$ As the boundaries of the right to health have expanded due to increased understanding of social determinants of health and the health impacts of the lived environment, legal frameworks have been insufficient in ensuring the protection of these rights. ${ }^{10}{ }^{11}$ Refugees are more likely to have poorer health during resettlement and face challenges in navigating legal, education, health, housing and employment services, which further threatens their quality of life and health status. ${ }^{12}$ A lack of coordination and integration across these services undermines service effectiveness. ${ }^{13}$

Much like the shift from the more vertical approaches of the millennium development goals towards the more integrated SDGs, the protection of the right to health too calls for an intersectoral approach whereby health is applied to all policies for all people. ${ }^{14}$ Therefore, for states to effectively protect the right to health for refugees, there is a need to work across sectors and disciplines to better integrate targeted programmes and initiatives, thereby improving standards of care during resettlement. Evidence exists that supporting collaboration and coordination across social services improves the quality of care received and its effectiveness. ${ }^{12}$ Furthermore, the refugee subpopulation is diverse and requires extraneous considerations in ensuring the right to health, as compared with the general population and also within the subpopulation itself. Many fragmented psychosocial programmes exist to attempt to address the unique challenges faced by refugees, but these are largely unevaluated and lack sustainability. ${ }^{15}{ }^{16}$ Better understanding, documentation and reporting of the dynamic nature of such interventions and their means of health system integration and intersectoral collaboration are necessary to ensure that lessons learnt are communicated and implemented in the design of future policies and programmes. This would promote continuity of care, people-centred care and sustainability of health and social services for refugees. Therefore, we aim to conduct a scoping review to explore barriers and facilitators to integrated health services for refugees; the content, process and actors involved in protecting refugee health; and the extent to which intersectoral approaches are leveraged to protect refugees' right to health on resettlement. This paper will outline the protocol for this review. The specific research questions for the review will be as follows:

1. What are the barriers and facilitators (context) in integrating targeted services for refugees within existing systems?

2. What strategies (content, process and actors) are involved in addressing refugees' right to health on resettlement?

3. To what extent are intersectoral approaches used to protect refugees' right to health, particularly in women and children?

\section{METHODS}

\section{Study design}

This study will be conducted using the scoping review methodology as described by the Joanna Briggs Institute Methods Manual for scoping reviews. ${ }^{17}$ Scoping reviews are used to map key concepts in an area to identify the scope of practice, working definitions, conceptual boundaries and the types of evidence available. We opted for a scoping review due to the complex nature of this topic, the changing global landscape around it and the insufficient evidence base to support effective decision making. ${ }^{18}$

The five stages outlined in a methodological framework for scoping studies are as follows: (1) identify the research question, (2) identify relevant studies, (3) study selection, (4) charting the data and (5) collating, summarising and reporting results. ${ }^{18}$

\section{Protocol}

The Preferred Reporting Items for Systematic Reviews and Meta-analysis for Protocols checklist were used in drafting this protocol. ${ }^{19}$

\section{Frameworks to address research questions}

While some evidence suggests that improved collaboration and coordination across social sectors can contribute to enhancing refugee health, there remains a need for a stronger evidence base on the context, processes and actors involved in protecting refugees' right to health on resettlement. ${ }^{12}$ Therefore, the research questions identified for this scoping review focus on integration and use of intersectoral approaches to address the complex needs of this vulnerable population. Two frameworks are being used concurrently in order to comprehensively identify barriers, 
facilitators, processes and actors involved at various stages in programme planning and implementation. The first is a framework for analysing integration of targeted health interventions in systems, where integration is defined as 'the extent, pattern, and rate of adoption and eventual assimilation of health interventions into each of the critical functions of a health system ${ }^{20}{ }^{20}$ Elements in this framework include (1) governance, (2) financing, (3) planning, (4) service delivery, (5) monitoring and evaluation (M\&E) and (6) demand generation. ${ }^{20}$ To be considered integrated, a health system intervention needs to fulfil certain requirements across these six areas as defined by the framework. ${ }^{20}$ We define an intervention here as changes in service delivery, organisational models, process modification, or new technologies. To satisfy governance needs for integration, governance and regulatory mechanisms for the intervention match those of the general health system. ${ }^{20}$ For financing, full integration has occurred when funding is incurred from national or regional budgets. ${ }^{20}$ In planning, which constitutes needs assessment, priority setting and resource allocation, full integration occurs when the same institutions and stakeholders are involved as those planning general health/other social systems. ${ }^{20}$ If service delivery is the responsibility of general staff embedded in the system, the intervention is considered integrated. ${ }^{20}$ Similarly, if M\&E was conducted by those with overall M\&E responsibility, then the intervention is considered integrated. ${ }^{20}$ Finally, demand generation is seen as integrated where services were promoted and incentivised by general staff within the existing system. ${ }^{20}$

The second framework applied is that of the Health in All Policies (HiAP) framework for country action. HiAP is defined as a way for countries to protect population health through 'an approach to public policies across sectors that systematically takes into account the health implications of decisions, seeks synergies, and avoids harmful health impacts in order to improve population health and health equity'. ${ }^{21}$ HiAP can be a powerful tool for enhancing accountability and safeguarding against distortions imposed by deleterious commercial and political interests. HiAP is in line with the human rights principles of legitimacy, protected by national and international law, accountability of governments to people, transparency of decision making, participation of the wider society, sustainability of policies to meet current needs without compromising future ones and collaboration across sectors and levels of government. ${ }^{1121}$ The HiAP framework for action involves six components including: (1) establish the need and priorities for HiAP, (2) frame planned action, (3) identify supportive structures and policies, (4) facilitate assessment and engagement, (5) ensure M\&E and (6) build capacity. ${ }^{21}$ These six components, adapted to refugee needs, will be used in the scoping review to frame barriers and facilitators in integrating refugee services across sectors through intersectoral collaboration. The framework for integration will then be used to assess the extent to which provisions for protecting refugees' right to health are integrated into existing social systems, and the content, process and actors involved in integration. ${ }^{22}$

\section{Identifying relevant studies}

Population

Eligible studies and papers will include those targeting refugees and asylum seekers as previously defined. We are not including other categories of migrants as their legal entitlements are different to those of refugees that are protected under international law. Asylum seekers have yet to be granted full legal refugee status as their request for sanctuary is still in process; however, we are including them in this review in order to capture any programmes that are also targeting this vulnerable group and reflect on differences in access to health across the two different stages of being granted sanctuary. This is especially relevant due to the scale of the current refugee crisis and the time it takes to be granted refugee status.

\section{Intervention}

Eligible studies and papers will describe a programme, approach or technical innovation that aims to protect refugees' right to health, including interventions aimed at addressing the social determinants of health. Interventions outside of the health sector that affect health will be included. If the studies do not display some level of integration or intersectorality, based on the frameworks for integration and HiAP, they will not be assessed further. ${ }^{20} 21$ This will be determined using a data abstraction chart where the key elements of the two frameworks will be laid out and contrasted against the studies found.

\section{Comparators}

Eligible studies and papers do not necessarily have to display a comparator as this scoping review is meant to gauge the state of the evidence. Where comparators exist, any types are relevant for inclusion, for example, those comparing a parallel approach to service provision for refugees versus an integrated approach.

\section{Outcomes}

Eligible studies and papers will include those discussing plans for action, strategies, barriers, facilitators or outcomes in integrating refugee health using an integrated or intersectoral approach. Studies or commentaries that solely discuss theories and conceptual models will be excluded.

\section{Study design}

Qualitative, quantitative and mixed methods will be eligible for inclusion. Experimental designs, including randomised controlled trials, non-randomised controlled trials and quasiexperimental models will be included, as well as observation and qualitative studies including cohort, cross-sectional, case series, ethnographic, interview-based and focus group discussion-based studies. Grey literature that explores plans, strategies, barriers, facilitators or outcomes of refugee health, as well as implementation research and operations research studies will also be included. Studies or report outlining stakeholder experiences and plans will also be included as case studies. 


\section{Time period}

In order to ensure relevance, only studies from 2000 onwards (search completed 8 May 2017) have been included, making the study period range over 16 years. It is estimated that the review will be completed by January 2018.

\section{Setting}

Eligible studies will be set in countries receiving refugees and asylum seekers (who may eventually qualify for refugee status) and serving as hosts for resettlement.

\section{Information sources and search strategy}

Based on the study team's concepts for the review, an experienced team of librarians from Karolinska Institutet have conducted a search of articles from 2000 to May 2017 in the following electronic databases: MEDLINE, Web of Science, Global Health and PsycINFO. The three concepts used to create the search strategy include: (1) refugees and asylum seekers; (2) type of service provision (health sector service delivery, intersectoral approaches, partnerships and integration); and (3) health equity, human rights and social determinants of health. See online supplementary appendix 1 for search strategy.

The search of the peer-reviewed literature will be supplemented by a search of grey literature through government websites, particularly governments of countries that receive the highest refugee burden, reports from multinationals and non-governmental organisations, conference abstracts, dissertations and news articles. Any additional report and articles will be identified by reaching out to relevant stakeholders in the authors' professional networks, including those involved in the European refugee response.

\section{Study selection process}

Search results will be cleaned for duplicates and uploaded to an Excel document, which will be used for screening using the eligibility criteria described above.

Two members of the study team will screen results based on the screening tool discussed. Inter-rater reliability will be assessed based on a set of 100 initial screens, and adjustments and clarifications to the screening tool will be made if reliability is not as high as desired (above $80 \%$ ). Once a set of included studies and papers are identified, two reviewers will independently conduct a full-text screen in order to apply the aforementioned frameworks for integration and intersectorality (HiAP). Eligible studies will be those displaying integration or intersectorality, defined as satisfying at least two of the six elements in either one of the integration or HiAP frameworks. A third reviewer has been identified in the event of disagreement between the two reviewers. This will be followed by data abstraction, using a form derived from the two framework, from the finalised set.

\section{Data abstraction and charting process}

General data collected will include study design, setting and journal discipline. Demographic data collected will include context, target study group (gender, age, ethnic background and status), number of participants, economic status of setting based on World Bank classifications and level of the health system where applicable (community, district, regional and so on). Intervention-specific data collected will include the type of intervention (behavioural, medical and social), the social determinant of health being addressed (WHO commission on social determinants of health framework), ${ }^{23}$ the primary sector(s) involved (health, education, law enforcement, housing and so on), duration, resources, funding source and conceptual framework applied if any. Outcome data collected will include barriers, facilitators, strategies, plans, qualitative findings from interviews and focus groups and any unintended consequences. Programmes and approaches specific to refugees versus asylum seekers will be disaggregated and distinctions highlighted. In the final set of included studies-those displaying some level of integration or intersectorality-key elements will be charted according to the two frameworks described above. ${ }^{182021}$ Data will be charted to include types of stakeholders involved in (1) governance, (2) financing, (3) planning, (4) service delivery, (5) M\&E and (6) demand generation. ${ }^{20}$ This will assess the extent of integration while data charted against the six components of the HiAP framework will assess the intersectoral potential of the intervention. Two members of the study team will code studies on NVivo software ${ }^{24}$ using a coding guide based on the two frameworks used for the review.

The data abstraction form will be tested by both reviewers using five studies. Where there is a sufficient level of agreement across reviewers (above 80\%), data abstraction will continue as designed. If agreement falls below the required range, the data abstraction form will be clarified. As this is a scoping review, meta-analysis will not be conducted.

\section{Risk of bias assessment}

In line with the manual used to design this scoping review, risk of bias assessments will not be conducted. ${ }^{17}$

\section{RESULTS}

Frequency tables will be used to describe included studies quantitatively, while narratives will be used to describe interventions, barriers, facilitators and other qualitative outcomes. Stakeholders will be presented based on the combined integration and HiAP frameworks, with their roles and involvement in the studies outlined. If a sufficiently diverse range of studies are identified, stratification by health system level (community, district and region) and country economy status will be done.

Charted data will be mapped out into subcategories to allow for a narrative description of barriers and facilitators, including barriers specific to vulnerable groups (women, children, torture survivors, those with disabilities and so on). New themes will be added where necessary, and elements of integration and/or intersectorality that are more or less prevalent across included studies will be 
highlighted. Finally, context, content, process and actors will be mapped based on charted data in accordance with the Walt Policy Triangle. ${ }^{22}$ Vulnerabilities of specific groups such as women and children will be highlighted.

\section{DISCUSSION}

\section{Implications}

This scoping review will identify programmes, approaches and interventions both within and outside the health sector that promote and protect refugees' right to health directly or indirectly through social determinants of health. To support country-level decision making and resettlement efforts, this review will provide an understanding of the extent of integration and intersectoral collaboration currently reported in this area, barriers and facilitators to provision of such services and their integration, and key stakeholders involved as well as those often missing. Findings will be shared with WHO colleagues working in this area, the Alliance for Health Policy and Systems Research contacts and a network of policy makers who will in turn share with their national and local networks. Other expected outputs include an improved understanding of contextual factors that are necessary in supporting the right to health for refugees as well as a narrative exploration of whether intersectoral collaboration and the idea of 'Health in All Policies' also works to protect and promote the health of persons outside of the traditional definitions of citizenry. These discussions will stimulate dialogue on how receiving countries can strengthen the resilience of their social systems to enhance their capacity for effective resettlement and improved health outcomes in their refugee populations.

\section{Ethics and dissemination}

In addition to the study team's respective networks, this review will also be disseminated at relevant conferences, meetings and communities of practice focused on enhancing use of evidence in policy making. A brief of key learnings will be created to support evidence-informed decision making in this area.

Ethics approval is not required for this scoping review as human subjects are not involved.

Acknowledgements The authors would like to thank Karolinska Institutet librarians, Magdalena Svanberg and Gun Brit Knutssön, for their contributions, specifically in running the search and identifying appropriate databases. We are also grateful to WHO Euro, specifically Dr Santino Severoni and Sara Barragan Montes, for their discussions and suggestions in the early stages of this project.

Contributors DJ drafted the paper and will be acting as primary reviewer; EVL, SH, PF and GT provided feedback and comments. SH and EVL will be acting as secondary reviewers. PF and GT of SIGHT will be providing continued support and connecting to relevant actors working in this area.

Funding In-kind time contributions from staff at the Alliance for Health Policy and Systems Research and Swedish Institute for Global Health Transformation, SIGHT, through support from the Bill and Melinda Gates Foundation, have made this possible.

Competing interests None declared.
Provenance and peer review Not commissioned; externally peer reviewed.

Open Access This is an Open Access article distributed in accordance with the Creative Commons Attribution Non Commercial (CC BY-NC 4.0) license, which permits others to distribute, remix, adapt, build upon this work non-commercially, and license their derivative works on different terms, provided the original work is properly cited and the use is non-commercial. See: http://creativecommons.org/ licenses/by-nc/4.0/

(C) Article author(s) (or their employer(s) unless otherwise stated in the text of the article) 2017. All rights reserved. No commercial use is permitted unless otherwise expressly granted.

\section{REFERENCES}

1. OHCHR. The Right to Health Geneva. Switzerland: Office of the United Nations High Commissioner for Human Rights \& WHO, 2008.

2. Tangcharoensathien V, Mills A, Palu T. Accelerating health equity: the key role of universal health coverage in the sustainable development goals. BMC Med 2015;13:101.

3. Rechel B, Mladovsky P, Ingleby D, et al. Migration and health in an increasingly diverse Europe. Lancet 2013;381:1235-45.

4. Langlois EV, Haines A, Tomson G, et al. Refugees: towards better access to health-care services. Lancet 2016;387:319-21.

5. UNHCR. Convention and Protocol relating to the status of refugees. Geneva, Switzerland: United Nations High Commissioner for Refugees, 2010.

6. UNHCR. UNHCR viewpoint: 'Refugee' or 'migrant' - Which is right? UNHCR. Geneva, Switzerland, 2016.

7. EU Cot. Council Directive 2003/9/EC of 27 January 2003 laying down minimum standards for the reception of asylum seekers. Official Journal of the European Union 2003:L31/18.

8. discrimination Coteor. General Recommendation 30: Discrimination against non-citizens. 2004: Committee on the elimination of racial discrimination, 2004

9. Norredam M, Mygind A, Krasnik A. Access to health care for asylum seekers in the European Union--a comparative study of country policies. Eur J Public Health 2006;16:285-9.

10. Jackson Bowers IC. Meeting the primary health care needs of refugees and asylum seekers. 16: PHCRIS Research Roundup, 2010.

11. WHO. Advancing the right to health: the vital role of law. Geneva, Switzerland: World Health Organization, O'Neil Institute, International Development Law Organization, University of Sydney, 2017.

12. Joshi C, Russell G, Cheng $I H$, et al. A narrative synthesis of the impact of primary health care delivery models for refugees in resettlement countries on access, quality and coordination. Int $J$ Equity Health 2013;12:88.

13. Protection DolaB. Fact sheet 60 - Australia's refugee and humanitarian program. Canberra: Australian Government: Department of Immigration and Border Protection, 2010.

14. UN. Integrated approaches to sustainable development planning and implementation. New York, USA: United Nations, 2015.

15. Kett ME. Internally displaced peoples in Bosnia-Herzegovina: impacts of long-term displacement on health and well-being. Med Confl Surviv 2005;21:199-215.

16. Patel N, Kellezi B, Williams AC. Psychological, social and welfare interventions for psychological health and well-being of torture survivors. Cochrane Database Syst Rev 2014;11:CD009317.

17. Micah Peters CG, Mclnerney P, Soares C, et al; The joanna briggs institute reviewers' manual: methodology for JBI scoping review. South Australia: Joanna Briggs Institute, 2015.

18. Arksey H, O'Malley L. Scoping studies: towards a methodological framework. Int J Soc Res Methodol 2005;8:19-32.

19. Moher D, Shamseer L, Clarke M, et al. Preferred reporting items for systematic review and meta-analysis protocols (PRISMA-P) 2015 statement. Syst Rev 2015;4:1.

20. Atun R, de Jongh T, Secci F, et al. Integration of targeted health interventions into health systems: a conceptual framework for analysis. Health Policy Plan 2010;25:104-11.

21. WHO. Health in all policies: framework for country action. France: World Health Organization, Finland Ministry of Social Affairs and Health, 2014.

22. Walt G, Shiffman J, Schneider H, et al. 'Doing' health policy analysis: methodological and conceptual reflections and challenges. Health Policy Plan 2008;23:308-17.

23. WHO. A conceptual framework for action on the social determinants of health. Geneva: WHO, 2010.

24. NVivo. NVivo 10. Australia: International QSR, 2012. 